\title{
Comparing Bacterial Leakage of Three Intraorifice Barrier Sealing Materials against Enterococcus faecalis and Proteus \\ vulgaris
}

Shreya Kohli Khanna ${ }^{1}$, H Murali Rao ${ }^{2}$, Nausheen Aga $^{3}$, Padma Chandra ${ }^{4}$

\begin{abstract}
Aim: The purpose of this in vitro study was to evaluate the intraorifice sealing ability of light-cured glass-ionomer cement (LC-GIC), Tetric N-Flow, and ProRoot mineral trioxide aggregate (MTA) against Enterococcus faecalis and Proteus vulgaris.

Materials and methods: Crowns of the eighty human mandibular teeth were decapitated. Working length determination was performed, after which cleaning and shaping were carried out. A uniform orifice diameter of $1.3 \mathrm{~mm}$, at its widest point, was made. Once instrumentation was completed, the canals were irrigated and then obturated. A heat carrier was used to remove gutta-percha to the depth of $3.5 \mathrm{~mm}$. Samples were then divided into a control group (Group 1) with no barrier, and three groups, namely, Group 2, Group 3, and Group 4, were restored with the LC-GIC, Tetric N-Flow, and ProRoot MTA, respectively. The groups were further subdivided into Subgroup A for checking bacterial leakage against $E$. faecalis and Subgroup B, against $P$. vulgaris. All samples were subjected to the bacterial leakage test and observed daily for the appearance of turbidity after which statistical analysis was performed.

Results: Group 1 showed leakage in, as early as, 3 days. The longest time for the turbidity to appear was shown by Group 4 with an average of 31 days. The mean number of days for turbidity to appear in Group 2 and Group 3 was 23 and 24 days, respectively. Group 4 showed the best intraorifice sealing ability with a significant difference.

Conclusion: The teeth with an intraorifice coronal seal had better protection against microbial leakage. Among all materials used, the ProRoot MTA showed the best intraorifice sealing ability.

Clinical significance: Use of the ProRoot MTA promises long-term results in the endodontically treated teeth as compared with other materials. Keywords: E. faecalis, Intraorifice barrier, LC-GIC, P. vulgaris, ProRoot MTA, Tetric N-Flow.

The Journal of Contemporary Dental Practice (2021): 10.5005/jp-journals-10024-3128
\end{abstract}

\section{INTRODUCTION}

The key purpose of root canal sealing is to stop any exchange between the nasal, root, and periradicular tissues and to thus prevent canal infection and reinfection. The deposit of residual microorganisms and irritants in the root canal system may directly affect the clinical result. One of the major factors associated with the endodontic failure ${ }^{1}$ is the microbial pollution of treated pulp space. Swanson and Madison reported that exposure to artificial saliva in coronal segments of shutting root canals results in 79 to $85 \%$ recontamination in less than 3 days. ${ }^{1}$ Several materials and procedures to avoid coronary microleakage have been suggested to date. The coronary restructuring of bacteria and their products after endodontic therapy can inhibit movement. The use of a material to screen the orifice could also avoid this bacterial penetration, in addition to a three-dimensional apical filling. ${ }^{2}$ Carmen and Wallace provide bacteria with a second line of support, according to Roghanizad and Jones, after endodontic treatment using intraorifice barrier products and the adhesive system's screening of pulp chambers. ${ }^{3}$ A successful alternative means of minimizing coronal leakage of endodontic treatment of the teeth is the intraorification membrane. The technique involves the placement, directly after the elimination of the coronal component of gutta-percha and the sealant, of additional materials into the channel openings. ${ }^{4}$ Various materials, such as amalgam, cavit, ionomer cement, composite, intermediate restaurating material (IRM), etc., were used as intraorific barriers to the prevention of coronary coronal
${ }^{1}$ MDS, Conservative Dentistry and Endodontics, Tutor, Government Dental College, Jamnagar, Gujarat

${ }^{2}$ DA Pandu Memorial RV Dental College, Bengaluru, Karnataka, India

${ }^{3}$ Department of Endodontics, University of Sharjah, Sharjah, United Arab Emirates

${ }^{4}$ Jawaharlal Medical College and Hospital, Bhagalpur, Bihar, India

Corresponding Author: Shreya Kohli Khanna, MDS, Conservative Dentistry and Endodontics, Tutor, Government Dental College, Jamnagar, Gujarat, e-mail: drshreyakl@gmail.com

How to cite this article: Khanna SK, Rao HM, Aga N, et al. Comparing Bacterial Leakage of Three Intraorifice Barrier Sealing Materials against Enterococcus faecalis and Proteus vulgaris. J Contemp Dent Pract 2021;22(6):674-679.

Source of support: Nil

Conflict of interest: None

microleakage in the radius of the root canal, but most of these materials display vulnerabilities (light-cured glass-ionomer cement $[\mathrm{LC}-\mathrm{GIC}])$. The mineral trioxide aggregate (MTA) was introduced as a bioactivity material in endodontic applications in the early 1990s. It is made of Portland Type I cement and consists of silica of dicalcium, silica of tricalcium, aluminium alloy, aluminoferrite of tetracalcium, and oxide of bismuth. It has several medicinal uses such as pulp caping, pulpotomy, internal root resorption 
treatment, undeveloped apices (apexogenesis and apexification), root-end refilling, root repair, and furcation perforations and coronary barrier. It also has many therapies. The resin-modified glass ionomer cement (RMGIC) in the late 1980s incorporates components of methacrylate which are common to all composites in resin. Over the years, it has shown superior performance as an acceptable coronary seal. Besides having a high bending strength and elasticity module, the excellent efficiency of the RMGIC can be clarified by the water sorption through the material, contributing to the expansion of water and hence a stronger seal. ${ }^{5}$ Flowable composites are composite resins with low viscosity that make them more fluid than typical composite resins. Tetric N-Flow is a flowing nanohybrid, light-curing, and radiopaque compound. It is claimed that they have higher ripple volumes, better tolerance to the inner cavity wall, faster alignment, and more versatility. For regular anterior and posterior restorations, the universal hybrid composite products offered the best general combination of strong material properties and clinical performance. ${ }^{6}$ The dye penetration procedure, electrochemical leakage tests, and liquid filtration technique are the most commonly used techniques in the evaluation of microleakage. The drawback of the dye penetration technique is that air inside the root canal filling can impede the penetration of the dye and thus impact the outcome when three analyses of leakage through the canal system and better simulate clinical scenarios are performed in a bacterial leakage assessment. ${ }^{7}$ Therefore, in this review, the effectiveness of sealing has been tested by bacterial leakage. The species used in the test of bacterial leakage were Enterococcus faecalis and Proteus vulgaris. E. faecalis is a nonmotile, spherical bacterium which is gram-positive. It is an optional anaerobic material of fermenting metabolism. E. faecalis has often been retrenched from apical periodontitis root canals and is involved in secondary infections as a significant endodontic pathogen. ${ }^{8}$ P. vulgaris is a that is mobile, gram-negative, and rodformed bacterium, which is viable for long periods in simple media. The root canal has been used as the most moving bacteria which penetrates easily from coronal to apical and leaks. ${ }^{9}$ The research aims to determine the effectiveness of intraorifice barrier materials for the Tetric N-flow, LC-GIC, and ProRoot MTA through the E. faecalis and $P$. vulgaris bacterial leakage testing.

\section{Materials and Methods}

This research was carried out in the Department of Conservative Dentistry and Endodontics, DA Pandu Memorial RV Dental College, Bengaluru. Eighty premolar human mandibular teeth extracted for periodontal and orthodontic purposes were considered, and in compliance with the recommendation and instructions from the Occupational Safety and Health Administration and the Center for Disease Control, they were stored, disinfected, and treated. The selection criteria of the teeth considered for the study were that they were not decayed and were single-rooted. Firstly, organic debris were removed by submerging the teeth in $1 \%$ sodium hypochlorite solution for 4 days followed by mechanical removal of calculus by ultrasonic scaling. The teeth were stored in $10 \%$ formalin for 2 weeks as per the standard sterilization protocol. After sterilization, the samples were stored in distilled water until further use.

The sample size has been estimated with the G Power v. 3.1.9.2 program. Given the calculation of the impact size (f) by $58 \%$, research strength by $80 \%$, and error margin by $5 \%$, the overall sample size required is 80 . These 80 teeth were further divided into four groups:
- Group 1: Control Group-This group had no intraorifice barrier material.

- Group 2: LC-GIC-LC-GIC was mixed in accordance with the manufacturer's instructions and restored into the teeth using a plastic filling instrument after which it was light cured.

- Group 3: Tetric N-Flow-Tetric N-Flow was used in accordance with the manufacturer's instructions and restored into the teeth using Teflon-coated instruments after which it was light cured.

- Group 4: ProRoot MTA-The ProRoot MTA was used in the channel using an amalgam carrier, and then condensed with a condenser in accordance with the manufacturer's instructions. The groups were further subdivided into Subgroup A for checking bacterial leakage against $E$. faecalis and Subgroup B, against $P$. vulgaris. Thus the groups and subgroups were divided as given in Figure 1.

The specimens were disinfected as per the universal sterilization protocol. These teeth were then stored in distilled water throughout the study. At the cementoenamel junction, the crowns of the harvested teeth were decapitated using diamond discs at a sluggish velocity. Channels were accessed, and a $10 \mathrm{~K}$ file was inserted into the channel before the file on the apical third is accessible. By extracting $1 \mathrm{~mm}$ of the registered length, the working length was calculated. The flaring method was performed using the ProTaper SX file (Denstply). Between the tools, $5.25 \%$ of sodium hypochlorite ( $\mathrm{NaOCl}$ ) and the RC-Prep technique were used. In a crown-down technique, the ProTaper SI, S2, FI, F2, and F3 files were used series, as instructed by the producer. The \#5 Gates Glidden bur was rendered to a depth of $3.5 \mathrm{~mm}$ with a uniform aperture diameter of $1.3 \mathrm{~mm}$ at its broadest point. The channels were rinsed after instrumentation, with $2 \mathrm{~mL}$ of $5.25 \% \mathrm{NaOCl}, 2 \mathrm{~mL}$ of $17 \%$ Ethylenediaminetetraacetic acid solution, distilling water, and final rinse of $0.2 \% \mathrm{NaOCl}$ chlorhexidine. The channels were sterilized with paper points and then sealed in lateral compaction techniques using F3 gutta-percha and zinc oxide eugenol sealer. The heat carrier was used for the removal of intestinal percha to $3.5 \mathrm{~mm}$ deep and a periodontal probe tested for depth.

The bacterial leakage test was carried out in which 2-week samples were stored with a relative humidity of $100 \%$ at $37^{\circ} \mathrm{C}$ to ensure that the entire collection of materials was usable. All the experimental teeth were coated with a three-layer nail varnish, from the root apex to the cement junction stage. The coronal part of each tooth was sealed with cyanoacrylate into the presterilized centrifuge lumen. This polyethene tube with a smaller diameter was sealed with cyanoacrylate in the upper half of the large presterilized vaccine. In the case of sterile condition, $P$. vulgaris was placed in the coronal reservoir of every assembly in the subgroup, was placed under sterilized condition of 0.20 to $01.25 \mathrm{~mL}$, and was then injected into the presterilized test tube of $3 \mathrm{~mL}$ of sterile Tryptic Soy Broth (TSB). In addition, it is also included. E. faecalis, rather than $P$. vulgaris, was used in Brain Heart Infusion (BHI) broth in Subgroup A. The test pipe was then vertically adjusted to racks, subsequently incubated at $37^{\circ} \mathrm{C}$ and observed daily for turbidity to occur in TSB and $\mathrm{BHI}$ bread apical reserves respectively. One $\mathrm{mL}$ of suspension (BHI and TSB) was sucked from the chamber every third day and substituted with $1 \mathrm{~mL}$ of the fresh sample. The bacterial growth resulting from the penetration of bacteria past the root canal was reported every day, demonstrating that the samples were observed for the turbidity of the bubble in the lower chamber. The medium was grown on a sterile swab or a blood agar medium, and after 24 hours, colonies were tested and stained with gram method of 


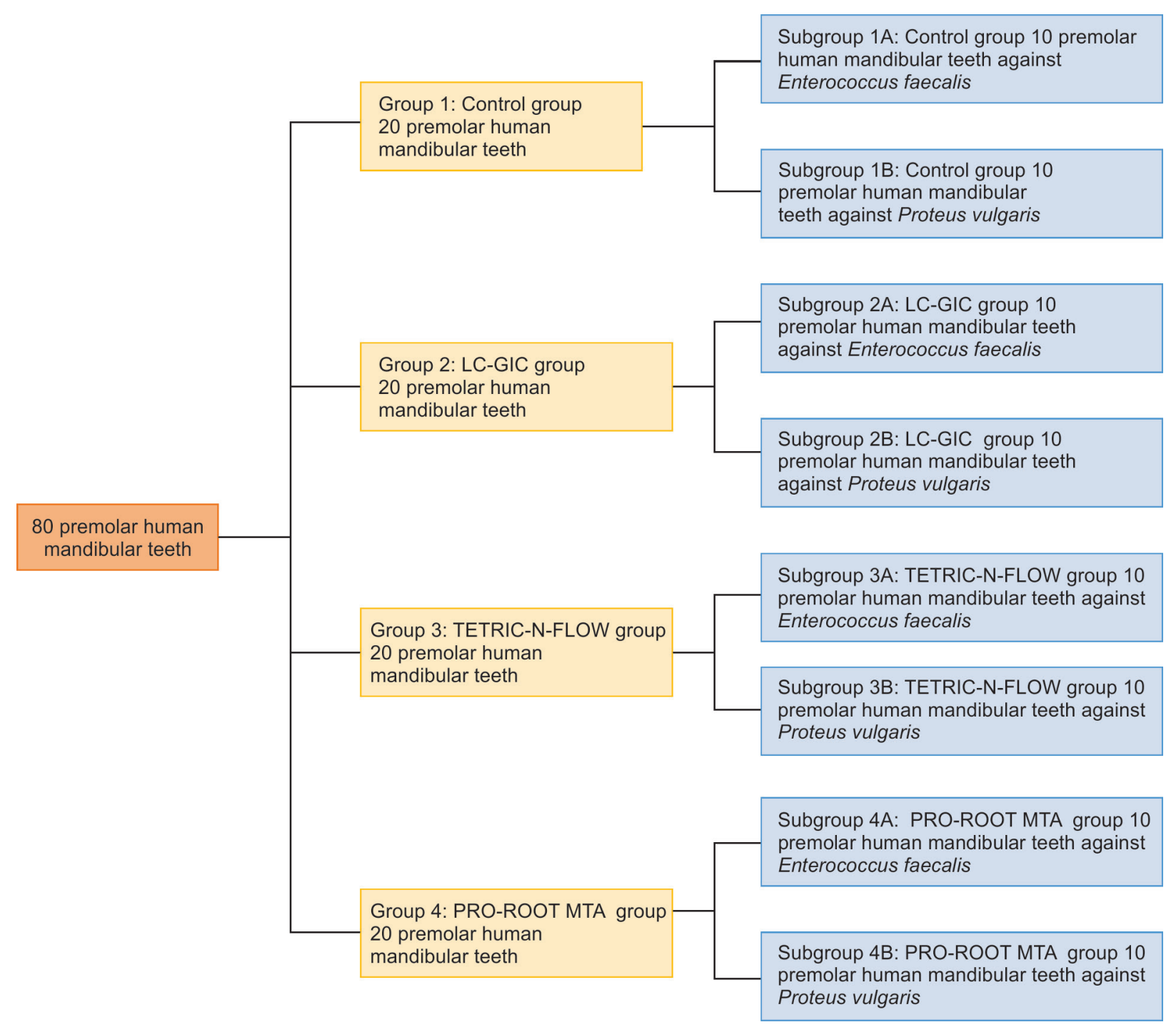

Fig. 1: Groups and subgroups of the study

staining and confirmed. The medium is grown with a sterile swab on a blood agar medium.

\section{Statistical Analysis}

A one-way analysis of variance (ANOVA) test followed by Tukey's honestly significant difference (HSD) post hoc analysis was used to compare the mean number of days taken to observe turbidity with $E$. faecalis and $P$. vulgaris between the study groups. The Student paired $t$ test was used to compare the mean number of days taken to observe turbidity between $E$. faecalis and $P$. vulgaris in different study groups.

The level of significance [ $p$ value] was set at $p<0.05$.

\section{Results}

In the present study, Group 1A showed leakage as early as 3.5 days. The longest time for the turbidity to appear was shown by Group 4A with an average of 31.75 days. The mean number of days for turbidity to appear in Group 2A and Group 3A was 23.3 and 24 days, respectively, as shown in Table 1. Table 2 represents Group 1A which showed statistically significant difference than Group 2A, Group 3A, and Group 4A with the $p$ value $<0.001$. The difference was found be statistically insignificant between Group $2 \mathrm{~A}$ and Group $3 \mathrm{~A}$ as the $p$ value was 0.46 . It was statistically significant between Group $2 A$ and Group 4A and also between Group 3A and Group 4A as the $p$ value was less than 0.001. Table 3 represents Group 1B which showed leakage in as early as 3.5 days. The longest time for the turbidity to appear was seen in Group 4B with an average of 31.38 days.The mean number of days for turbidity to appear in Group 2B and Group 3B was 22.4 and 23.5 days, respectively. Table 4 represents Group $1 B$ showed statistically significant difference than Group 2B, Group $3 \mathrm{~B}$, and Group 4B with $p$ value less than 0.001 . The difference was found to be statistically insignificant between Group 2B and Group $3 \mathrm{~B}$ with the $p$ value 0.07 . It was statistically significant between Group 2B and Group 4B and also between Group 3B and Group 4B with the $p$ value less than 0.001 . Table 5 shows that the difference was statistically insignificant between Group 1 and Group 4. It was statistically significant in Group 2 and Group 3 with a $p$ value of 0.004 and 0.02 , respectively. Considering the results, it can be concluded that ProRoot MTA provides a significantly better coronal seal than LC-GIC and Tetric N-Flow in the endodontically treated teeth. 
Table 1: Comparison of the mean number of days taken to observe for turbidity with respect to $E$. faecalis between the study groups using the one-way ANOVA test followed by Tukey's HSD post hoc analysis

\begin{tabular}{lrrllcccc}
\hline Groups & $N$ & Mean & Standard deviation & Std.error & Minimum & Maximum & Factor & $p$ value \\
\hline Control & 10 & 3.50 & 0.53 & 0.17 & 3 & 4 & & \\
LC-GIC & 10 & 23.30 & 1.25 & 0.40 & 21 & 25 & & \\
$\begin{array}{l}\text { Tetric } \\
\text { N-Flow }\end{array}$ & 10 & 24.00 & 0.82 & 0.26 & 23 & 25 & 1218.34 & $<0.001^{*}$ \\
$\begin{array}{l}\text { ProRoot } \\
\text { MTA }\end{array}$ & 8 & 31.75 & 1.49 & 0.53 & 29 & 33 & & \\
\hline
\end{tabular}

"Significance

Table 2: Multiple comparison of mean difference in the number of days for turbidity from $E$. faecalis between groups using Tukey's HSD post hoc test

\begin{tabular}{|c|c|c|c|c|c|}
\hline \multirow[b]{2}{*}{ Group (I) } & \multirow[b]{2}{*}{ Group $(J)$} & \multirow[b]{2}{*}{ Mean diff } & \multicolumn{2}{|c|}{$\begin{array}{l}\text { 95\% Confidence } \\
\text { intervals for the } \\
\text { diff }\end{array}$} & \multirow[b]{2}{*}{$p$ value } \\
\hline & & & Lower & Upper & \\
\hline \multirow{3}{*}{ Control } & LC-GIC & -19.80 & -21.08 & -18.52 & $<0.001^{*}$ \\
\hline & Tetric N-Flow & -20.50 & -21.78 & -19.22 & $<0.001^{*}$ \\
\hline & ProRoot MTA & -28.50 & -29.61 & -26.89 & $<0.001^{*}$ \\
\hline \multirow{2}{*}{ LC-GIC } & Tetric N-Flow & -0.70 & -1.98 & 0.58 & 0.46 \\
\hline & ProRoot MTA & -8.45 & -9.81 & -7.09 & $<0.001^{*}$ \\
\hline Tetric N-Flow & ProRoot MTA & -7.75 & -9.11 & -6.39 & $<0.001^{*}$ \\
\hline
\end{tabular}

*Significance
Table 4: Multiple comparisons of the mean difference in the number of days for turbidity from P.vulgaris between groups using the Tukey's HSD post hoc test

\begin{tabular}{|c|c|c|c|c|c|}
\hline \multirow[b]{2}{*}{ Group (I) } & \multirow[b]{2}{*}{ Group $(J)$} & \multirow[b]{2}{*}{ Mean diff } & \multicolumn{2}{|c|}{$\begin{array}{c}95 \% \text { Confidence } \\
\text { intervals for } \\
\text { the diff }\end{array}$} & \multirow[b]{2}{*}{$p$ value } \\
\hline & & & Lower & Upper & \\
\hline \multirow{3}{*}{ Control } & LC-GIC & -18.90 & -20.06 & -17.74 & $<0.001^{*}$ \\
\hline & Tetric N Flow & -20.00 & -21.16 & -18.84 & $<0.001^{*}$ \\
\hline & ProRoot MTA & -27.86 & -29.10 & -26.65 & $<0.001^{*}$ \\
\hline \multirow{2}{*}{ LC-GIC } & Tetric N-Flow & -1.10 & -2.26 & 0.06 & 0.07 \\
\hline & ProRoot MTA & -8.98 & -10.20 & -7.75 & $<0.001^{*}$ \\
\hline Tetric N-Flow & ProRoot MTA & -7.88 & -9.10 & -6.65 & $<0.001^{*}$ \\
\hline
\end{tabular}

${ }^{*}$ Significance

Table 3: Comparison of the mean number of days taken to observe for turbidity with respect to $P$. vulgaris between the study groups using the one-way ANOVA test followed by Tukey's HSD post hoc analysis

\begin{tabular}{lrrllllll} 
Groups & $N$ & Mean & Standard deviation & Std. error & Minimum & Maximum & $F$ & pvalue \\
\hline Control & 10 & 3.50 & 0.53 & 0.17 & 3 & 4 & & \\
LC-GIC & 10 & 22.40 & 1.17 & 0.37 & 21 & 25 & & \\
Tetric & & & & & & & $1424.794<0.001^{*}$ \\
N-Flow & 10 & 23.50 & 0.85 & 0.27 & 22 & 25 & & \\
$\begin{array}{l}\text { ProRoot } \\
\text { MTA }\end{array}$ & 8 & 31.38 & 1.19 & 0.42 & 30 & 33 & & \\
\hline
\end{tabular}

*Significance

Table 5: Comparison of the mean number of days taken to observe for turbidity between $E$. faecalis and P.vulgaris in different study groups using the Student Paired $t$ test

\begin{tabular}{lllllllll}
\hline Group & Organisms & $N$ & Mean & Standard deviation & SEM & Mean diff & $t$ & $p$ value \\
\hline \multirow{2}{*}{ Control } & E. faecalis & 10 & $3.50^{\mathrm{a}}$ & 0.53 & 0.17 & \multirow{2}{*}{0.00} & - & - \\
& P. vulgaris & 10 & $3.50^{\mathrm{a}}$ & 0.53 & 0.17 & & & \\
\multirow{2}{*}{ LC-GIC } & E. faecalis & 10 & 23.30 & 1.25 & 0.40 & 0.90 & 3.857 & $0.004^{*}$ \\
& P. vulgaris & 10 & 22.40 & 1.17 & 0.37 & & & \\
\multirow{2}{*}{ Tetric N-Flow } & E. faecalis & 10 & 24.00 & 0.82 & 0.26 & & \\
& P. vulgaris & 10 & 23.50 & 0.85 & 0.27 & & 3.000 & $0.02^{*}$ \\
ProRoot MTA & E. faecalis & 8 & 31.75 & 1.49 & 0.53 & & & \\
& P. vulgaris & 8 & 31.38 & 1.19 & 0.42 & 0.38 & 1.158 & 0.29 \\
\hline * & & & & & & & &
\end{tabular}

*Significance

alt's level of significance in ANOVA Test 


\section{Discussion}

The research in vitro compared intraorifice barriers to the coronal microskid-containing MTA mixture, cement enriched by calcium (calcium-enriched mixture [CEM]), and biodentine. The microleakage was least seen by CEM cement as an intraorifice barrier in endodontically treated teeth..$^{10}$ In the intracoronal bleaching, Moghadam et al. ${ }^{11}$ compared the scale properties of the CEM with that of the MTA, In the evaluation of the sealing capability of the agents and the sealing capacity of MTA and CEM cement, a process of colour penetration has been used. The measurement of the ionomer and the ProRoot MTA for light-curing microleakage was conducted with coronal barriers in intracoronal bleaching for endodontically treated teeth. During bleaching, the MTA delivered a stronger coronal seal. ${ }^{12}$ A research was carried out to determine the impact on the effectiveness of endodontic therapy of coronal restoring consistency and root filling. Demographic statistics, including evaluations of the coronation filling (type, quality), radical or coronar fractory, and the periodontal condition around a dent, were registered, and clinical reports of patients treated at Dental Clinics University were published from 2003 to 2014 of patients treated at Kuwait University Dental Clinics (KUDC). The health of the periapical tissues was determined with the periapical index by means of an elevated radiograph of the endodontal tooth. The root filling efficiency was measured using the radiation filling length and density. The median time of follow-up was 4.8 years. The coronary microleakage of MTAs, CEM), and intraorifice barrier biodentine was contrasted with the least microleakage in the endodontically treated teeth in this in vitro study. ${ }^{10}$ The sealing qualities of the CEMs were contrasted with those of MTAs as cervical barrier in the process of intracoronal bleaching by Moghadam et al. In addition to the intraorification barrier of $3 \mathrm{~mm}$ and sodium perborate mixture, the treatment of endodontics by CEM cement and MTAs was used. The system used for colour penetration has served to determine agents' scale capacity, and there has been a substantial ignorance to the scale capacity of MTA and CEM cements. ${ }^{11}$ Assessing microleakage as a coronary challenge for the intracoronal bleaching of endodontically treated teeth was performed for light-healed glaze ionomers and ProRoot mineral trioxides. During bleaching, the MTA delivered a stronger coronal seal. ${ }^{12}$

The sealing capability of glass ionomer cement (GIC) as coronal screening materials for light healing composite and light healing was compared and evaluated. The findings revealed the increase in coronal sealing capability for light cure GIC.13 A research was carried out to determine the impact on the effectiveness of endodontic therapy of coronal restoring consistency and root filling. Demographic statistics, including evaluations of the coronation filling (type, quality), radical or coronar fractory, and the periodontal condition around a dent, were registered and clinical reports of patients treated at KUDC were published from 2003 to 2014. The research has indicated a higher incidence of periapical lesions but not the qualitative nature of root filling. ${ }^{14}$

Different materials were used to create a coronal seal (cavit, medium-term restore, ionomer cement, zinc oxide eugenic cement, composites, etc.); each content has its own advantages and restrictions. While studies confirm the efficacy of coronal intraorifice obstacles, there is no universal protocol that included a coronary barrier after root canal therapy. Therefore, this research was carried out in the bacterial system against E. faecalis and $P$. vulgaris, testing the intraorific sealing capacity of LC-GIC, Tetric N-flow, and MTAs. Through evaluating the findings of this analysis, a statistical discrepancy between the groups studied was found. In the control group which had no barrier in both bacterial samples, the microleakage was observed during the minimum 3.5-day period. This can also be viewed as an indication of leakage potentiality and is compatible with studies by Jones et al. and Swanson et al. which have shown that gutta-percha and sealer do not provide a sufficient seal, implying the use of intraorifice barriers for better sealing. The longest time for the turbidity to appear was shown by Group 4 (ProRoot MTA) with an average of 31 days. The mean number of days for turbidity to appear in Group 2 (LC-GIC) and Group 3 (Tetric N-Flow) was 23 and 24 days, respectively. Group 4 showed the best intraorifice sealing ability with a significant difference. Group 2 and Group 3 had a significant difference with the control group but were statistically insignificant among themselves. It was found that both LC-GIC and Tetric N-flows were higher than controls, but there was no statistically meaningful distinction, as was the case with studies by Shetty et al. and Parolia et al. ${ }^{15,16}$ Owing to their control layout and improved adhesion to the tooth structure, LC-GIC was chosen. The better sealing capacity of LC-GIC can be ascertained by forming an ion-exchange layer next to dentin and the shear bond strength of LC-GIC which, by two methods, acid base reaction common to all glass ionomer and photochemical polymerization of water soluble monomers and methacrylate groups, is greater than traditional GIC, RMGICs. ${ }^{17}$ The shrinkage of polymerization of these materials continues to take place largely because of the resin portion, but immature cement still uses dentine fluid which expands the material, which may compensate for the shrinkage of the polymerizing. ${ }^{15}$ In the present analysis, samples with the ProRoot MTA displayed the highest turbidity average time as the barrier to the two bacterial classes optimum screening potential. The Portland concrete, bismuth oxide, and other metal oxides trace quantities are compounded with the MTA. The biocompatibility with superior leakage tolerance is outstanding in both the short and long term. It extends the layout and thereby leads to a strong seal. This expansion is expected to lead to a superior scale potential and superior marginal change in the MTA-resistant leakage. It was used as a root filler during apical surgery and as an apical plug in open, nonvital apex teeth and now as a coronal barrier. ${ }^{18}$ This is used for vital pulp therapy and the reconstruction of fural and lateral perforations. The findings of this research are related to previous studies by Yavari et al. ${ }^{4}$ and Shetty et al. ${ }^{16}$ Roghanizad and Jones research results showed the use of amalgam in the prevention of coronal microleak, like an orifice plug, than Cavit. The sealing capacity between the MTA, composite, or cavit has been unreported to Sanchez et al. ${ }^{19}$ Reports of Jenkins et al. ${ }^{20}$ have been based on a review. The MTA, an intraorifice barrier that is compatible with the findings of the analysis, is significantly stronger than Tetric. Based on the recent analysis, Sanchez et al. stated that during the testing time, CavitTM G, Tetric EvoFlowor, and ProRoot MTA had similar resistance skills to leaks when they were used as intraorifice barriers. ${ }^{18}$ BarrieshiNusair and Hammod compared glass ionomer and the MTA as plugs and recorded more microleak. ${ }^{21}$ The proposed procedure for an immediate coronary seal remains feasible and has several benefits. The coronal $3 \mathrm{~mm}$ channel is a perfect narrow cavity that can easily be screened and is surrounded by an intact teeth structure. In this perfect cavity, there are no occlusal or chewing powers. This approach does not have any esthetic concerns because the substance is intracanally located. However, more 
caution is required on the anterior teeth, as the suggested substance can induce tooth coloration or affect potential bonding agents typically used in these teeth. More research is required on this topic. The 3-screen depth of the cavity indicated has two advantages: (a) it contains ample scanning material for adequate screening. A $3.5 \mathrm{~mm}$ material thickness was proven to be sufficient for a decent dressing. (b) Removability should be required reasonably and quickly removed. The approach proposed is simple and effective. The cavity is dry and isolated, with the rubber dam still on; a heated unit is separated from the gutta-percha $3.5 \mathrm{~mm}$ and sealed. No extra time or special equipment is needed for this. In this study, single-rooted premolar teeth with single-patent root canal were chosen to reduce the anatomical heterogeneity and to achieve standardization. In this study, single-rooted premolar teeth with single-patent root canal were selected. The test root canal samples were decoronated before the root canal instrumentation. This was performed to avoid the variance of access cavity preparations and helps for the easy placement of the syringe needle during irrigation and obturation and get standardized intraorifice barriers for all the samples. The highest aversion time in the MTA was observed in the present study, and the lowest time was observed in the supportive control group, which indicates an average of 3.5 days. The MTA showed the best results owing to its superior sealing ability and marginal adaptation. Tetric and LC-GIC followed this with no significant difference among them. In contrast with the teeth without a coronal seal during the test era, the teeth with a coronal seal had greater protection against microbial leakage. This proves intraorifice barriers as an effective measure to prevent microleakage and the ProRoot MTA as a promising material as an intraorifice barrier.

\section{Limitations of the Study}

The study has two limitations. First being that more in vivo studies are required to compare various techniques and material to check bacterial leakage. Secondly, more studies are required to check the long-term effectivity of these materials against $E$. faecalis and $P$. vulgaris after obturation with gutta-percha.

\section{Conclusion}

It can be inferred within the limits of this in vitro analysis that the intraorifice material serves as an efficient microleak shield. The ProRoot MTA provides a significantly better coronal seal than LC-GIC and Tetric N-Flow in endodontically treated teeth thus providing long-term results in clinical patients after endodontic therapy.

\section{References}

1. Swanson K, Madison S. An evaluation of coronal microleakage in endodontically treated teeth. Part I. Time periods. J Endod 1987;13(2):56-59. DOI: 10.1016/S0099-2399(87)80155-3.

2. Parekh B, Irani RS, Sathe S, et al. Intraorifice sealing ability of different materials in endodontically treated teeth: an in vitro study. J Conserv Dent 1996;17(3):234-237. DOI: 10.4103/0972-0707.131783.

3. Roghanizad N, Jones J. Evaluation of coronal microleakage after endodontic treatment. J Endod 1996;22(9):471-473. DOI: 10.1016/ S0099-2399(96)80080-X.
4. Yavari HR, Samiei M, Shahi S. Microleakage comparison of four dental materials as intra-orifice barriers in endodontically treated teeth. Iran Endod J 2012;7(1):25-30.

5. Cattani-Lorentea MA, Dupuisb V, Payanc J, et al. Effect of water on the physical properties of resin-modified glass ionomer cements. Dent Mater 1999;15(1):71-78. DOI: 10.1016/s0109-5641(99)00016-0.

6. Aboobaker S, Nair BG, Gopal R, et al. Effect of intra-orifice barriers on the fracture resistance of endodontically treated teeth - an ex-vivo study. J Clin Diagn Res 2015;9(2):17-20. DOI: 10.7860/ JCDR/2015/11609.5552.

7. Samiei M, Aghazade M, Farhadi F, et al. Sealing efficacy of single-cone obturation technique with MTA and CEM cement: an in vitro bacterial leakage study. J Dent Res Dent Clin Dent Prospects 2014;8(2):77-83. DOI: $10.5681 /$ joddd.2014.014.

8. Delboni MG, Gomes BPFA, Francisco PA, et al. Diversity of Enterococcus faecalis genotypes from multiple oral sites associated with endodontic failure using repetitive sequence-based polymerase chain reaction and arbitrarily primed polymerase chain reaction. J Endod 2017;43(3):377-382. DOI: 10.1016/j.joen.2016.10.042.

9. Jacob S, Kumar R, Narayanan L. Evaluation of microleakage with and without smear layer: a bacterial penetration study. J Endod 2000;12(2):77-82.

10. Ramezanali F, Aryanezhad S, Mohammadian F, et al. In vitro microleakage of mineral trioxide aggregate, calcium-enriched mixture cement and biodentine intra-orifice barriers. Iran Endod J 2017;12(2):211-215. DOI: 10.22037/iej.2017.41.

11. Moghadam N, Abdollahi AA, Aghabalayi Fakhim H, et al. In vitro sealing properties of calcium-enriched mixture and mineral trioxide aggregate orifice barriers during intra-coronal bleaching. Iran End J 2017;12(2):231-235. DOI: 10.22037/iej.2017.45.

12. Jahromi MZ, Barekatain M, Hashemi NB, et al. Assessment of micro-leakage for light-cure glass ionomer and pro-root mineral trioxide aggregate as coronal barriers in intracoronal bleaching of endodontically treated teeth. Caspian J Dent Res 2017;6(1):22-28. DOI: 10.22088/cjdr.6.1.22.

13. Jaiswal $P$, Jain A, Motlani M, et al. Comparative evaluation of sealing ability of light cure glass ionomer cement and light cure composite as coronal sealing material: an in vitro study. J Int Clin Dent Res Org 2017;9(1):12. DOI: 10.4103/2231-0754.203047.

14. Maslamani M, Khalaf M, Mitra AK. Association of quality of coronal filling with the outcome of endodontic treatment: a follow-up study. Dent J 2017;5(1):5. DOI: 10.3390/dj5010005.

15. Parolia A, Kundabala M, Acharya S,et al. Sealing ability of four materials in the orifice of root canal systems obturated with gutta-percha. Endodontology 2008;20(2):65-70.

16. Shetty A, Srinivasan R, Nasreen F. Comparison of the sealing ability of mineral trioxide aggregate and three conventional restorative materials when placed coronally over gutta-percha as sealing materials-an in vitro study. Endodotology 2010;22:15-21.

17. Mavec JC, McClanahan SB, Minah GE, et al. Effects of an intracanal glass ionomer barrier on coronal microleakage in teeth with post space. J Endod 2006;32(2):120-122. DOI: 10.1016/j.joen.2005.10.033.

18. Metgud SS, Shah HH, Hiremath HT, et al. Effect of post space preparation on the sealing ability of mineral trioxide aggregate and gutta-percha: a bacterial leakage study. J Conserv Dent 2015;18(4):297-301. DOI: 10.4103/0972-0707.159729.

19. Bailón-Sánchez ME, González-Castillo S, González-Rodríguez MP, et al. Intraorifice sealing ability of different materials in endodontically treated teeth. Med Oral Patol Oral Cir Bucal 2011;16(1):105-109.

20. Jenkins S, Kulild J, Williams K, et al. Sealing ability of three materials in the orifice of root canal systems obturated with gutta-percha. JOE 2006;32(3):225-227. DOI: 10.1016/j.joen.2005.10.042.

21. Agarwal RS, Hiremath $\mathrm{H}$, Agarwal J, et al. Evaluation of cervical marginal and internal adaptation using newer bulk fill composites: an in vitro study. J Conserv Dent 2015;18(1):56-61. DOI: 10.4103/0972-0707.148897. 